\title{
Strong enhancement of forbidden atomic transitions using plasmonic nanostructures
}

\author{
A. M. Kern* and O. J. F. Martin ${ }^{\dagger}$ \\ Nanophotonics and Metrology Laboratory (NAM), Swiss Federal Institute of Technology (EPFL), CH-1015 Lausanne, Switzerland
}

(Received 25 March 2011; revised manuscript received 25 October 2011; published 1 February 2012)

\begin{abstract}
We investigate the mediation of symmetry-forbidden atomic transitions using plasmonic nanostructures. We show that the excitation of the electric dipole-forbidden, quadrupole-allowed $6^{2} S_{1 / 2}-5^{2} D_{5 / 2}$ transition in cesium may be enhanced by more than 6 orders of magnitude in the intense, inhomogeneous near field of a plasmonic nanoantenna. Using optical reciprocity, the enhancement can be understood to apply to spontaneous emission as well, allowing the fast and efficient optical detection of excited atoms.
\end{abstract}

DOI: 10.1103/PhysRevA.85.022501

PACS number(s): 32.70.Cs, 78.67.-n, 32.80.-t, 73.20.Mf

\section{INTRODUCTION}

In recent years, plasmonic nanostructures have been used extensively for enhancing fluorescence and Raman scattering at surrounding molecules. Astounding single-molecule detection has been demonstrated experimentally, displaying both high sensitivity and spacial selectivity [1-7]. These feats are possible due to the ability of plasmonic particles to concentrate light into strongly localized and enhanced hot spots. These subwavelength modes allow selective probing of single molecules, the field enhancement increasing fluorescence or scattering intensities to a level which can more easily be measured.

The coincidence of high intensity and localization also supports the enhancement of another type of process. The high field inhomogeneity observed in the near field of nanoscopic objects can be used to excite dipole-forbidden atomic transitions that are nearly unobservable under planewave illumination [8]. In combination with the high field enhancement observed in their near field, plasmonic nanostructures display field gradients unmatched by those obtained using conventional methods such as tight focusing or evanescent fields; thus they should be ideally suited for mediating symmetry-forbidden processes. In this work, the mediation of electric dipole-forbidden, quadrupole-allowed transitions using a plasmonic nanoantenna is investigated in a case study of the $6^{2} S_{1 / 2}-5^{2} D_{5 / 2}$ transition in cesium. A quantitative expression for the enhancement in an arbitrary field is derived to then be applied to the numerically calculated field around a nanoantenna. The plasmonically enhanced field is shown to boost absorption and spontaneous emission of the quadrupole transition by 6 orders of magnitude, much more than dipole-allowed transitions, for which enhancement is in the hundreds [9].

\section{METHOD}

When exciting an atomic transition with an external electromagnetic field, the transition rate $\gamma$ can be derived from Fermi's golden rule using time-dependent perturbation

\footnotetext{
*Current address: Institute of Physical and Theoretical Chemistry, Eberhard Karls University, 72076 Tübingen, Germany; a.kern@uni-tuebingen.de

†olivier.martin@epfl.ch
}

theory [10] and we can write $\gamma \propto\left|\left\langle\varphi_{f}|W| \varphi_{i}\right\rangle\right|^{2}$. Here, $W$ is the Hamiltonian for the interaction of an electron with the external electromagnetic field and $\varphi_{i}$ and $\varphi_{f}$ are the electronic wave functions of the transition's initial and final states, respectively. The general form of $W$ neglecting spin is

$$
W=-\frac{e}{m} \mathbf{p} \cdot \mathbf{A}+\frac{e^{2}}{2 m}|\mathbf{A}|^{2}+e \phi,
$$

where $e, m$, and $\mathbf{p}$ are the electron's charge, mass, and canonical momentum and $\mathbf{A}$ and $\phi$ are the electromagnetic vector and scalar potentials at the position of the electron. By appropriate choice of gauge, the potentials $\phi$ and $\mathbf{A}$ can be expressed in terms of Taylor expansions of the electric and magnetic fields ( $\mathbf{E}$ and $\mathbf{B})$ about the origin $(\mathbf{r}=\mathbf{0})$, i.e., the atom's nucleus [11], and the interaction Hamiltonian can be written as

$$
W=e \mathbf{r} \cdot \mathbf{E}_{0}-e \mathbf{Q}:(\nabla \mathbf{E})_{0}-e \mathbf{m} \cdot \mathbf{B}_{0}+\cdots,
$$

where $e \mathbf{r}$ is the electric dipole, $e \mathbf{Q}=\frac{e}{2} \mathbf{r r}$ is the electric quadrupole, and $e \mathbf{m}=\frac{e}{2 m} \mathbf{r} \times \mathbf{p}$ is the magnetic dipole moment. Here, $(\mathbf{C D})_{i j}=C_{i} D_{j}$ is the outer product; thus $\nabla \mathbf{F}$ is the transposed Jacobian matrix of $\mathbf{F}$. The subscript 0 denotes a value at the origin and the double dot product is defined as $\mathbf{C}: \mathbf{D}=\sum_{i j} C_{i j} D_{j i}$. In this study, the mediation of electric quadrupole transitions in a harmonic electromagnetic field is considered and so the corresponding transition rate can be described using

$$
\gamma_{Q}=C_{1}\left|\left\langle\varphi_{f}|\mathbf{Q}| \varphi_{i}\right\rangle:(\nabla \mathbf{E})_{0}\right|^{2},
$$

with the constant factor $C_{1}$.

The lower electronic states of alkali-metal atoms take on a simple form as their shell contains only one valence electron. The wave function of this electron can be separated into radial and angular parts,

$$
\varphi_{n l m}(r, \theta, \phi)=R_{n l}(r) Y_{l m}(\theta, \phi),
$$

where $n, l$, and $m$ are the principal, azimuthal, and magnetic quantum numbers, respectively. The function $R_{n l}$ is only a function of the radius and $Y_{l m}$ is spherical harmonics. Separating the $r$ dependence from the quadrupole moment, $\mathbf{Q}=r^{2} \frac{\mathbf{Q}}{r^{2}}$, the transition rate can be written as

$$
\gamma_{Q}=C_{2}\left|\left\langle Y_{l^{\prime} m^{\prime}}\left|\frac{\mathbf{Q}}{r^{2}}\right| Y_{l m}\right\rangle:(\nabla \mathbf{E})_{0}\right|^{2},
$$


where $C_{2}=C_{1}\left|\left\langle R_{n^{\prime} l^{\prime}}\left|r^{2}\right| R_{n l}\right\rangle\right|^{2}$. Here, the $r$ dependence is evaluated with the radial function $R_{n l}$, leaving $\frac{\mathbf{Q}}{r^{2}}$ to couple with the spherical harmonics [12]. The quantum numbers of the initial and final wave functions are written unprimed and primed, respectively.

The dipole-forbidden, quadrupole-allowed $6{ }^{2} S_{1 / 2}-$ $5^{2} D_{5 / 2}$ transition in cesium lends itself to the current study as its energy lies in the visible range at $\lambda=685 \mathrm{~nm}$. This transition was the subject of a theoretical and experimental study utilizing the inhomogeneous evanescent field at total internal reflection to mediate the otherwise nearly unobservable absorption [13]. The $5 d$ shell of cesium is initially empty, and so the transition rate must be summed over all $m^{\prime}=-2, \ldots, 2$. The matrices $\left\langle Y_{2 m^{\prime}}\left|\frac{\mathbf{Q}}{r^{2}}\right| Y_{00}\right\rangle$ can then easily be computed by integration over the solid angle. Finally, as the atom is randomly oriented, the transition rate is averaged over the orientation $[14,15]$. Note that for fixed atom alignment, the transition rate will depend on the magnetic quantum number $m^{\prime}$ of the final state [12]. Here, however, the atoms are randomly oriented and the spacial averaging yields identical transition rates for each $m^{\prime}$.

To calculate the transition enhancement in an inhomogeneous field, one first must calculate the transition rate under plane-wave illumination, for which the matrix $\nabla \mathbf{E}$ takes on a very simple form. For example, for an $x$-polarized plane wave propagating in the $z$ direction, $\mathbf{E}^{\mathrm{pw}}=E_{0} \hat{\mathbf{e}}_{x} e^{i k z}$, one obtains only one nonzero element, $\left(\boldsymbol{\nabla} \mathbf{E}^{\mathrm{pw}}\right)_{z x}=i k E_{0} e^{i k z}$, where $k$ is the norm of the wave vector. The orientation-averaged transition rate can then be computed to $\bar{\gamma}_{Q}^{\mathrm{pw}}=C_{2} k^{2}\left|E_{0}\right|^{2} / 15$. Considering that we have averaged over the orientation, this result is valid for plane waves of all polarization and propagation directions. One can now define the enhancement factor $F_{Q}$ of an arbitrary field as the enhancement of the transition rate compared to that obtained under plane-wave illumination, $F_{Q}=\bar{\gamma}_{Q} / \bar{\gamma}_{Q}^{\mathrm{pw}}$. The constant $C_{2}$ then cancels out and does not need to be determined.

For certain inhomogeneous fields, the Jacobian matrix can be analytically computed. For example, the evanescent field of a plane wave totally internally reflected at an interface takes on a well-known form [12,13]. For sake of comparison, the system described in Ref. [13] was reproduced with the formulation given in this paper, assuming $\lambda=685 \mathrm{~nm}$ and a glass prism with $n=1.456$ in vacuum. For the simpler case of $s$ polarization, this leads to the expected enhancement of $F_{Q} \sim 3$ at the interface, decreasing with the distance from the glass surface.

When calculating the enhancement factor $F_{Q}$ around a plasmonic nanoantenna, the Jacobian matrix cannot be calculated analytically. Instead, surface integral equation (SIE) simulations [16] were used here to determine $\nabla \mathbf{E}$ numerically. As a geometry, gold dipole antennas with square cross sections of $40 \times 40 \mathrm{~nm}^{2}$ and gap sizes $g$ between 5 and $25 \mathrm{~nm}$ were chosen (see Fig. 1). The arm lengths were engineered so that all the antennas resonated at $\lambda=685 \mathrm{~nm}$, with the relative permittivity of gold $\epsilon_{r}=-15.4+1.02 i$ taken from Ref. [17]. The antennas were excited by $x$-polarized plane waves propagating along the $z$ axis. Difference quotients with steps of $0.1 \mathrm{~nm}$ were used to compute the spatial derivatives.

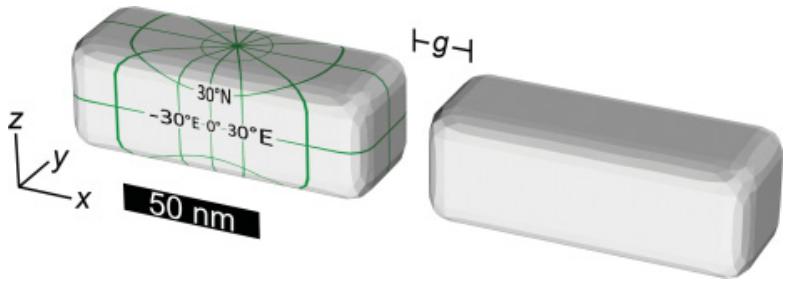

FIG. 1. (Color online) Geometry of the simulated nanoantennas. The gap width $g$ is varied between 5 and $25 \mathrm{~nm}$; the length of the $40 \times 40 \mathrm{~nm}^{2}$ cross section arms is varied so the antennas resonate at $\lambda=685 \mathrm{~nm}$.

\section{RESULTS AND DISCUSSION}

\section{A. Absorption enhancement}

First, the quadrupole enhancement was calculated at points $1 \mathrm{~nm}$ above the surface of the antennas' left arms. The obtained enhancement factors for resonant excitation are shown in Fig. 2 for gap sizes $g=5$ and $25 \mathrm{~nm}$.

The values are displayed using an equirectangular representation, unwrapped like a map of the globe (see Fig. 1). The arms' right (inside) and left (outside) ends are located at $\left(0^{\circ} \mathrm{N}, 90^{\circ} \mathrm{E}\right)$ and $\left(0^{\circ} \mathrm{N},-90^{\circ} \mathrm{E}\right)$, respectively. Many properties of the enhancement can be seen here. First, stronger enhancement can be obtained with a smaller gap size. This is in part because the field enhancement in the gap is greater for smaller gap sizes [18]. Increasing the total field strength will increase the elements of $\boldsymbol{\nabla} \mathbf{E}$ by the same degree, resulting in a higher enhancement factor $F_{Q}$. Second, a very small gap size will result in a nearly homogeneous field distribution in the gap, like that inside a plate capacitor. The elements of $\mathbf{\nabla E}$ should thus be small in the space between the antenna's arms, rising as one leaves the gap. This behavior is clearly visible

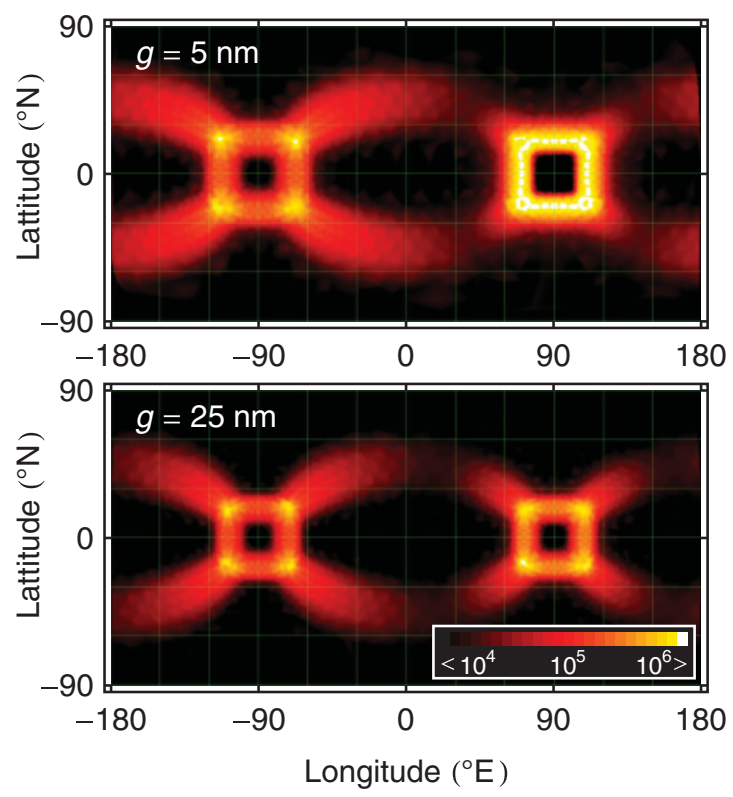

FIG. 2. (Color online) Quadrupole transition rate enhancement $F_{Q}$ at points $1 \mathrm{~nm}$ above the surface of the left arms of resonantly excited gold nanoantennas with gap sizes of $g=5$ and $25 \mathrm{~nm}$. Values are displayed using an equirectangular representation. 

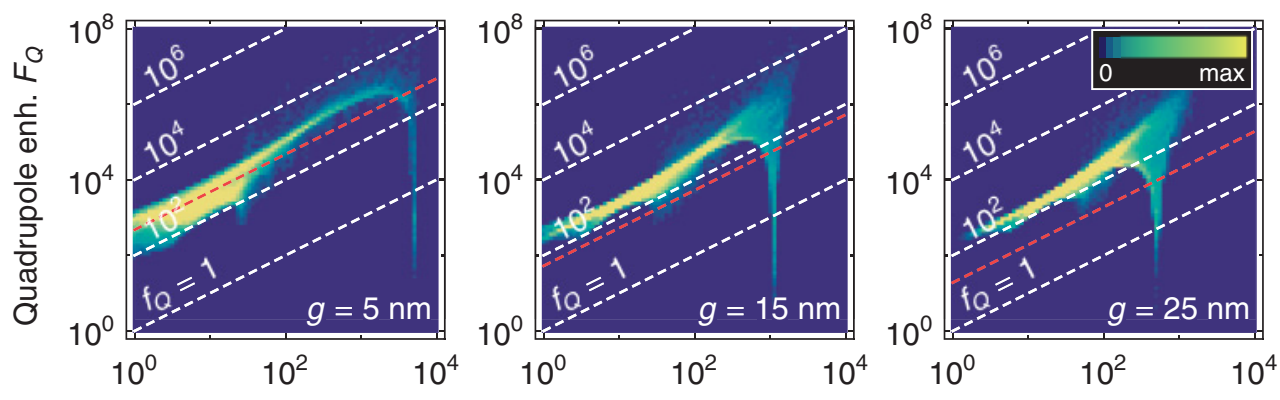

Intensity enhancement $F_{\text {int }}$

FIG. 3. (Color online) Log-axis histograms of the field in an $(80 \mathrm{~nm})^{3}$ box around the gaps of antennas with gap widths $g=5$, 15 , and $25 \mathrm{~nm}$. The white dashed lines show values of constant $f_{Q}=F_{Q} / F_{\text {int }}$ and the red dashed lines show the approximated $f_{Q}=(k g)^{-2}$ for a subwavelength aperture.

in Fig. 2, especially for $g=5 \mathrm{~nm}$. For larger gaps, or at the antennas' outside edges, the largest transition enhancement can be observed at the antennas' corners (rounded to an 8-nm radius in the simulations). Here, a high gradient in the field strength is expected due to the lightning-rod effect. Finally, one can see that enhancement factors of greater than $10^{6}$ are obtained, suggesting that a plasmonic antenna may indeed lead to a considerable and measurable increase in the transition rate.

To quantify the behavior in the three regions observable in Fig. 2 (far from the antenna, at the corners, and near the gap), the intensity and quadrupole enhancement factors were computed at $2 \times 10^{5}$ points in a box enclosing all three regions. Histograms of these calculations, shown in Fig. 3, allow one to identify and characterize the behavior in the different regions. Note that in this logarithmic representation, the histograms' bins scale with the axes, and so the graphs are more sensitive for higher axis values. The first region, far from the antenna, is represented by the points with low-intensity enhancement $\left(F_{\text {int }} \sim 10\right)$ and shows comparable behavior for the three shown gap widths. The field at the corners of the antennas is strongly enhanced and inhomogeneous, leading to large intensity and quadrupole enhancement factors, and is represented by the faint tails reaching upward of $F_{\text {int }}=10^{3}$ and $F_{Q}=10^{6}$. Finally, the region around the gap is characterized by a transition from a strong, homogeneous field in the gap (high $F_{\text {int }}$ and low $F_{Q}$ ) over a rapid field decay (lower $F_{\text {int }}$ and high $F_{Q}$ ) to continually decreasing intensity (decreasing $F_{\text {int }}$ and $F_{Q}$ ). This behavior is easily recognized in the curved lines beginning on the bottom right of the graphs (i.e., in the gap) and arcing up and to the left. These curves show a strong dependence on the gap width $g$. The largest quadrupole enhancement in the gap region can be extracted from the curves' maxima. For small gap widths, this maximum exceeds $10^{6}$ and overlaps the enhancement at the corners, in agreement with the behavior shown in Fig. 2 .

The histograms in Fig. 3 show that the intensity and quadrupole enhancement factors are correlated, as observed in Fig. 2. The contribution of the intensity enhancement can be removed from $F_{Q}$ by considering the quotient $f_{Q}=F_{Q} / F_{\text {int }}$, indicated by the white dashed lines in Fig. 3. This corresponds to the enhancement caused purely by the localization of the field around the antennas and the resulting field gradient, i.e., the local modification of the transition's oscillator strength.
With the exception of the region inside the gap, this factor takes on values between $10^{2}$ and $10^{4}$.

In the near field behind a subwavelength aperture, the wave vector can be approximated by $k^{\prime}=1 / w$, where $w$ is the width of the aperture [19]. By replacing $w$ with the antenna's gap width $g$, this leads to a value of $f_{Q}=(k g)^{-2}$ for the enhancement near the gap, indicated by the red dashed lines in Fig. 3. One can see that while the maximum simulated enhancement near the gap follows the trend given by this approximation, the values for the nanoantenna are in fact higher. This is explained by the fact that the antenna not only confines the light in its gap, but locally enhances its intensity there as well, leading to a higher field gradient even after division by $F_{\text {int }}$.

\section{B. Emission enhancement}

Until now, the excitation enhancement of quadrupole transitions was discussed. Using the theory of electromagnetic reciprocity [20,21], the enhancement can be interpreted to apply to spontaneous emission as well [9]. A large increase in the local density of optical states in the vicinity of the nanoantenna causes a decrease in the excited state's lifetime by the same factor as derived for the excitation enhancement. With enhancement factors of more than $10^{5}$, quadrupole transitions may show decay rates similar to or larger than those normally observed in dipole-allowed transitions in free space. One must take into account, however, that an atom decaying in the vicinity of a plasmonic structure may be quenched, decaying into a nonradiative mode, and the energy converted to heat by the losses in the metal [22]. To investigate the efficiency of the emission process, the behavior of a quadrupole emitter located in the midgap plane was investigated. Figure 4(a) shows the enhancement $F_{\text {rad }}$ of the power $P_{\text {rad }}$ radiated to the far field compared to free-space emission for quadrupole emitter positions between the gap's center $(z=0)$ and edge $(z=20 \mathrm{~nm})$, assuming a constant quadrupole moment. One can clearly see the reverse process of that shown in Fig. 2. The highest emission enhancement is reached not in the gap's center but when leaving the homogeneous field in the gap at around $z \approx 15 \mathrm{~nm}$. Calculating the power $P_{\text {abs }}$ absorbed by the gold nanoantenna, one can then compute the radiative quantum yield $Q=P_{\mathrm{rad}} /\left(P_{\mathrm{rad}}+P_{\mathrm{abs}}\right)$ of the emission process, shown in Fig. 4(b). A high quantum yield of $\sim 0.3$ is obtained 
(a)

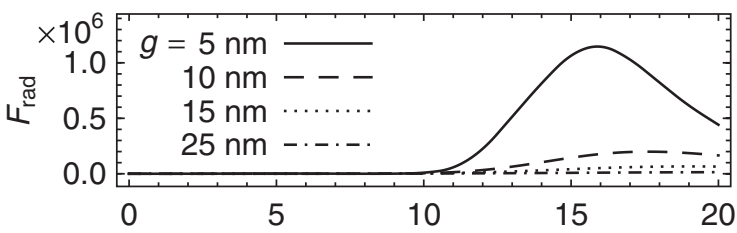

(b)

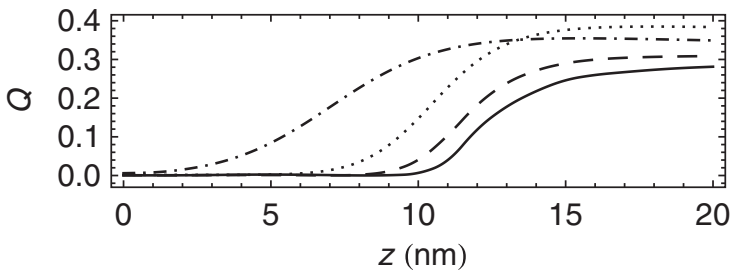

FIG. 4. Radiated power enhancement (a) and quantum yield (b) of an ideally oriented quadrupole emitter located in the midgap plane at distances $z$ from the gap's center.

at the position of maximum radiation. For a dipole emitter near a plasmonic particle, the shortest decay lifetime, obtained at the particle's surface, is annulled by a vanishing radiative quantum yield, resulting in nearly zero radiated power [22]. In the case of a quadrupole emitter decaying in the gap region of a nanoantenna, on the other hand, a strongly shortened lifetime is reached some distance from the antenna's surface. The resulting high quantum yield leads to a very large enhancement of the power radiated to the far field.

\section{Discussion}

In the last section it was shown that a plasmonic nanoantenna can enhance the transition rate of an atomic quadrupole transition by over 6 orders of magnitude-much more than in an evanescent field. This result is now compared to the effect of other nanoscale objects reported in the literature. For example, enhancement of quadrupole transitions in the near field of a dielectric nanosphere has been discussed in an interesting analytical investigation [8]. While the intensity enhancement around a dielectric sphere is expected to be less than the hot spots observed near plasmonic nanoparticles, the field gradient can still be large given a small enough sphere. However, to obtain the enhancement factor of $10^{6}$ observed for nanoantennas, a glass sphere would have to be about $1 \mathrm{~nm}$ in diameter, resulting in an extremely small enhancement volume. In addition, such a small sphere would be difficult to fabricate in a controlled geometric distribution, making selective enhancement at specific atoms problematic.

The quadrupole enhancement factor has also been calculated for subwavelength metallic gratings in a recent numerical study [19]. Apart from strong enhancement at the rectangular grating's corners, high values of $f_{Q} \sim 1000$ are only reported for nearly vanishing $F_{\text {int }}$ and thus low $F_{Q}$. A plasmonic nanoantenna, on the other hand, displays strong $f_{Q}$ and $F_{Q}$ in the region near the gap in addition to the large enhancement at the corners. Besides being tunable in enhancement factor and volume, the enhancement in the gap region has the advantage of being separated from the surface of the antennas, which reduces quenching during emission processes as discussed in Sec. III B.
The strong enhancement of an atom's radiative decay rate can be used for fast preparation and detection of longlived states. Approaching a trapped atomic cloud toward a nanostructured surface [23], selective interaction of atoms with plasmonic nanoantennas might be realized. This process could be implemented in a miniaturized atomic clock or in quantum computation applications, where preparation and detection of long-lived atomic states are required for the initialization and readout of computation processes. Efficient near-field coupling from the nanoantenna to a plasmonic waveguide [24] could then be used to directly pass along the quantum signal [25].

In the applications described above, it may be necessary to keep the used atoms at very low temperatures and thus a low kinetic energy. In this case, the comparatively weak forces on the atoms induced by nonresonant dipole interaction and the Casimir-Polder (CP) potentials must be considered [26] as they may prevent the atoms from entering the interaction region around the antennas. The CP force at a surface is generally attractive and will actually facilitate interaction, drawing atoms toward antennas located on the surface. A nonresonant dipole in an electric field gradient, on the other hand, can give rise to a force opposite (in) the direction of the field gradient for oscillating fields blue (red)-shifted with respect to the dipole's resonance frequency [27]. If an atom exhibits a strong electric dipole-allowed transition red-detuned with respect to the investigated quadrupole transition, the resulting force may thus prevent it from approaching the antenna. At room temperature, the kinetic energy of the atoms is much higher than the $\mathrm{CP}$ and nonresonant dipole potentials and these forces can be neglected.

Finally, the extremely high enhancement factor of the quadrupole transition rate leads to a large contrast in fluorescence between atoms close to a nanoantenna and those further away. While the enhancement of dipole-allowed transitions will provide a contrast of $\sim 5$, neglecting intrinsic quenching [22,28], a quadrupole transition will experience an enhancement of many orders of magnitude, matching the contrast of surface-enhanced Raman scattering, but with a larger initial cross section.

\section{SUMMARY}

In this paper, we have shown that symmetry-forbidden atomic transitions can be strongly enhanced using plasmonic nanostructures. As an example, a local enhancement by more than 6 orders of magnitude was calculated for the excitation of the $6^{2} S_{1 / 2}-5^{2} D_{5 / 2}$ electric quadrupole transition in cesium near a gold nanoantenna. This effect is stronger than reported for other nanostructures due to the capacity of the nanoantenna to both strongly increase and localize the field. The quadrupole enhancement applies to spontaneous emission as well, displaying a comparatively high quantum yield due to a separation of the enhancement region from the antenna's surface.

\section{ACKNOWLEDGMENT}

This work was supported by the Swiss National Science Foundation (Grant No. 20021-116758). 
[1] S. Nie and S. R. Emory, Science 275, 1102 (1997).

[2] K. Kneipp, Y. Wang, H. Kneipp, L. T. Perelman, I. Itzkan, R. R. Dasari, and M. S. Feld, Phys. Rev. Lett. 78, 1667 (1997).

[3] X. S. Xie and J. K. Trautman, Annu. Rev. Phys. Chem. 49, 441 (1998).

[4] A. J. Meixner, T. Vosgröne, and M. Sackrow, J. Lumin. 94-95, 147 (2001).

[5] J. Jiang, K. Bosnick, M. Maillard, and L. Brus, J. Phys. Chem. B 107, 9964 (2003).

[6] S. Kühn, U. Håkanson, L. Rogobete, and V. Sandoghdar, Phys. Rev. Lett. 97, 017402 (2006).

[7] J. A. Dieringer, R. B. Lettan, K. A. Scheidt, and R. P. Van Duyne, J. Am. Chem. Soc. 129, 16249 (2007).

[8] V. V. Klimov and V. S. Letokhov, Phys. Rev. A 54, 4408 (1996).

[9] A. M. Kern and O. J. F. Martin, Nano Lett. 11, 482 (2011).

[10] C. Cohen-Tannoudji, J. Dupont-Roc, and G. Grynberg, AtomPhoton Interactions (Wiley, Weinheim, 2004).

[11] L. D. Barron and C. G. Gray, J. Phys. A 6, 59 (1973).

[12] S. Tojo and M. Hasuo, Phys. Rev. A 71, 012508 (2005).

[13] S. Tojo, M. Hasuo, and T. Fujimoto, Phys. Rev. Lett. 92, 053001 (2004).

[14] D. L. Andrews and N. P. Blake, J. Phys. A 22, 49 (1989).

[15] J. A. Crosse and S. Scheel, Phys. Rev. A 79, 062902 (2009).
[16] A. M. Kern and O. J. F. Martin, J. Opt. Soc. Am. A 26, 732 (2009).

[17] P. B. Johnson and R. W. Christy, Phys. Rev. B 6, 4370 (1972).

[18] H. Fischer and O. J. F. Martin, Opt. Express 16, 9144 (2008).

[19] K. Deguchi, M. Okuda, A. Iwamae, H. Nakamura, K. Sawada, and M. Hasuo, J. Phys. Soc. Jpn. 78, 024301 (2009).

[20] L. Landau, E. Lifchitz, and L. Pitaevskii, Electromagnetics of Continuous Media (Pergamon, Oxford, 1984).

[21] E. L. Ru and P. Etchegoin, Chem. Phys. Lett. 423, 63 (2006).

[22] P. Anger, P. Bharadwaj, and L. Novotny, Phys. Rev. Lett. 96, 113002 (2006).

[23] C. Stehle, H. Bender, C. Zimmermann, D. Kern, M. Fleischer, and S. Slama, Nat. Photonics 5, 494 (2011).

[24] A. Alù and N. Engheta, Phys. Rev. Lett. 104, 213902 (2010).

[25] D. E. Chang, A. S. Sørensen, P. R. Hemmer, and M. D. Lukin, Phys. Rev. Lett. 97, 053002 (2006).

[26] H. Bender, P. W. Courteille, C. Marzok, C. Zimmermann, and S. Slama, Phys. Rev. Lett. 104, 083201 (2010).

[27] R. Grimm, M. Weidemüller, and Y. B. Ovchinnikov, Adv. At. Mol. Opt. Phys. 42, 95 (2000).

[28] O. L. Muskens, V. Giannini, J. A. Sanchez-Gil, and J. Gomez Rivas, Nano Lett. 7, 2871 (2007). 